\title{
Predictors of blood pressure response to ultrasound renal denervation in the RADIANCE-HTN SOLO study
}

\author{
Manish Saxena $\mathbb{1}^{1} \cdot$ Roland E. Schmieder (10) ${ }^{2}$ Ajay J. Kirtane ${ }^{3}$ Felix Mahfoud (10) ${ }^{4,5}$ - Joost Daemen ${ }^{6}$ Jan Basile ${ }^{7}$ \\ Philipp Lurz ${ }^{8}$ Philippe Gosse $\mathbb{1}^{9}{ }^{9}$ - Kintur Sanghvi ${ }^{10}$ - Naomi D. L. Fisher ${ }^{11}$ - Lars C. Rump ${ }^{12}$ - Atul Pathak ${ }^{13}$. \\ Peter J. Blankestijn ${ }^{14}$ - Anthony Mathur ${ }^{1}$ Yale Wang $\mathbb{D}^{15} \cdot$ Michael A. Weber $\mathbb{D}^{16} \cdot$ Andrew S. P. Sharp $^{17,18}$. \\ Michael J. Bloch ${ }^{19} \cdot$ Neil C. Barman $\mathbb{D}^{20} \cdot$ Lisa Claude ${ }^{20} \cdot$ Yang Song $^{21} \cdot$ Michel Azizi ${ }^{22,23,24} \cdot$ Melvin D. Lobo $\mathbb{B}^{1}$
}

Received: 5 January 2021 / Revised: 9 April 2021 / Accepted: 23 April 2021 / Published online: 24 May 2021

(c) The Author(s) 2021. This article is published with open access

\begin{abstract}
The blood pressure (BP) lowering response to renal denervation (RDN) remains variable with about one-third of patients not responding to ultrasound or radiofrequency RDN. Identification of predictors of the $\mathrm{BP}$ response to RDN is needed to optimize patient selection for this therapy. This is a post-hoc analysis of the RADIANCE-HTN SOLO study. BP response to RDN was measured by the change in daytime ambulatory systolic blood pressure (dASBP) at 2 months post procedure. Univariate regression was used initially to assess potential predictors of outcome followed by multivariate regression analysis. In the univariate analysis, predictors of response to RDN were higher baseline daytime ambulatory diastolic blood pressure (dADBP), the use of antihypertensive medications at screening, and presence of orthostatic hypertension (OHTN) whilst the presence of untreated accessory arteries was a negative predictor of response. Multivariate analysis determined that dADBP and use of antihypertensive medications were predictors of response to RDN with a trend for OHTN to predict response. Obese females also appeared to be better responders to RDN in an interaction model. RDN is more effective in patients with elevated baseline dADBP and those with OHTN, suggesting increased peripheral vascular resistance secondary to heightened sympathetic tone. These assessments are easy to perform in clinical setting and may help in phenotyping patients who will respond better to RDN.
\end{abstract}

\section{Introduction}

Novel antihypertensive therapies using second-generation renal denervation (RDN) catheter systems causing modification of renal sympathetic nerve signaling have shown encouraging results in randomized controlled trials [1-4]. The multicenter, randomized, double-blind, sham-controlled

Previous Presentations: Preliminary anatomic predictors (without clinical predictors), and preliminary clinical predictors (without anatomic predictors) were presented in abstracts at ESH2018 and TCT 2018. This article summarizes a new model that combines both anatomic and clinical predictors.

Supplementary information The online version contains supplementary material available at https://doi.org/10.1038/s41371021-00547-y.

Manish Saxena

m.saxena@qmul.ac.uk

Extended author information available on the last page of the article
RADIANCE-HTN SOLO study evaluated endovascular ultrasound RDN in an off-medication population with mild-moderate combined (systolic-diastolic) hypertension [1]. To date, results from the RADIANCE-HTN SOLO study have demonstrated the efficacy and safety of the Paradise System in lowering daytime ambulatory systolic blood pressure (dASBP) in patients with primary hypertension at 2 months (off-medication) and 6 months (on-medications) post-randomization $[1,5]$.

Given that RDN is invasive to perform compared to drug therapy, the lack of markers of procedural success is a significant issue at the present time [6,7]. Whilst ongoing studies are addressing potential biomarkers of successful renal nerve ablation, it would also be helpful to improve phenotyping to best determine responders/ non-responders to therapy given that in RADIANCEHTN SOLO approximately one-third of patients exhibited $<5 \mathrm{mmHg}$ reduction in dASBP in the treatment group at 2 months while remaining off-medications [1]. 
Previous studies have analyzed the clinical, anatomical, and procedural predictors of $\mathrm{BP}$ response to RDN [8-10]. Importantly, many of the predictors could be study specific based on factors such as the patient cohort included, study design, as well as procedural parameters including energy modality used (radiofrequency (RF) or ultrasound), number/ location of ablation sites, between-interventionalist variability. Herein for the first time, we aim to analyze clinical, anatomical, and procedural predictors of BP response in a trial specific to endovascular ultrasound RDN. We specifically focused on predictors which are usually associated with sympathetically driven hypertension including higher basal heart rate [11], orthostatic hypertension [12-14], and abdominal obesity [15].

\section{Methods}

This is a post-hoc analysis of the RADIANCE-HTN SOLO study data. To summarize, RADIANCE-HTN SOLO was a multicenter, international, double-blind, randomized, shamcontrolled trial done at 21 centers in the USA and 18 centers in Europe [1]. The study was approved by local ethics committees at each center, and all participants provided written informed consent for participation in the trial.

\section{Patients}

Male or female patients aged 18-75 years with combined systolic-diastolic hypertension (office BP $\geq 140 / \geq 90 \mathrm{mmHg}$ ) on 0-2 antihypertensive medications were enrolled in the study. Patients were eligible for randomization if they had daytime ambulatory $\mathrm{BP}(\mathrm{ABP}) \geq 135 / \geq 85 \mathrm{mmHg}$ and $<170 /$ $<105 \mathrm{mmHg}$ after a 4-week discontinuation of their antihypertensive medications and had suitable renal artery anatomy on CT- or MR-angiography.

\section{Trial procedure}

Eligible patients were randomized in a 1:1 ratio to either the sham procedure (only renal angiography) or ultrasound RDN with the Paradise catheter System (ReCor Medical, Palo Alto, CA, USA) after renal angiography under conscious sedation. Patients, outcome assessors, and study personnel were blinded to the randomization. Patients were not started on any antihypertensive medications for 2 months post procedure unless specified BP escape criteria were met.

Patients were assessed at 2 months post procedure for evaluation of the primary efficacy endpoint which was change in daytime ambulatory systolic BP (dASBP) from baseline in the intention-to-treat population. Major adverse events and safety outcomes were also collected.
The study is registered with ClinicalTrials.gov, number NCT02649426.

\section{Office BP measurements}

Office BP was recorded using the Omron ${ }^{\circledR}$ MIT ELITE Plus or M10-IT device (Omron Co., Kyoto, Japan). All efforts were made to standardize office BP recordings for each patient including measurement from the same arm at same time of day using the same device by the same person. Caffeine, exercise, and smoking were avoided for at least 30 min prior to the measurement and patients sat quietly in a chair for at least $5 \mathrm{~min}$ before recordings.

An appropriately sized cuff was used to ensure the bladder within the BP cuff encircled at least $80 \%$ of the arm. BP was measured in both arms and the arm with the higher reading was used for office BP recording. For study defined BP measurements, three sitting $\mathrm{BP}$ and heart rate (HR) measurements were recorded, 1-2 min apart. The average of the 2nd and the 3rd measurements have been used for analysis. Standing BP and HR were recorded after 1 min to check the presence of OHTN, defined as office standing minus office seated systolic blood pressure $(\mathrm{SBP}) \geq 20 \mathrm{mmHg}$ and/or office standing minus office sitting diastolic blood pressure $(\mathrm{DBP}) \geq 10 \mathrm{mmHg}$ at baseline. The presence of any symptoms was not required for the diagnosis of OHTN.

\section{Ambulatory BP measurements}

The 24-h ambulatory $\mathrm{BP}$ (24-H ABP) recordings were made using the Microlife ${ }^{\circledast}$ WatchBP machine (Microlife, Taipei, Taiwan) at baseline and 2-month follow-up visits. The ambulatory blood pressure (ABP) was recorded on patient's non-dominant arm for $25 \mathrm{~h}$. BP was recorded every $20 \mathrm{~min}$ during daytime (07:00-22:00 h) and every $30 \mathrm{~min}$ overnight (22:00-07:00 h). 24-H ABP recordings with a minimum of 21 measurements during the daytime period were considered valid. In the case of a non-valid measurement, a new ABP recording was performed. All ABP recordings were sent to a core laboratory (dabl, Dublin, Ireland) with treatment assignments masked.

\section{Statistical analysis}

All analyses were conducted on the per-protocol population $(\mathrm{RDN}=64$ and Sham $=58)$. As previously described, the per-protocol population excluded patients who did not meet baseline daytime ambulatory SBP or DBP or renal artery anatomical inclusion criteria, patients in the RDN group who did not receive bilateral RDN, patients who were treated with antihypertensive medications before the 2-month 24-H ABP, and patients who did not complete the 2-month 24-H ABP assessment [1]. 
Univariate regression was used to assess potential predictors of outcome defined as change in dASBP at 2 months. In addition, we evaluated whether predictors of outcome differed between responders (defined as reduction in $\mathrm{dASBP} \geq 5 \mathrm{mmHg}$ ) and super responders (defined as reduction in dASBP $\geq 15 \mathrm{mmHg}$ ) at 2 months following treatment.

Multivariate regression analysis was conducted for the RDN arm including variables with $p$ value less than 0.20 from the univariate analysis. Backward selection with a stay criterion of 0.20 was used to select predictors. In the multivariate regression analysis, we checked that the variance inflation factor was less than 5 for all input variables.

Multiple interaction analyses were conducted looking at the impact of these variables: treatment (RDN vs. Sham), sex (male vs. female), abdominal obesity (yes vs. no), and age $(<55$ vs. $\geq 55)$ on change in dASBP. The least square means were used to compare the effect size of different combinations.

Treatment effects (change in dASBP parameters, HR) were assessed comparing patients with and without orthostatic hypertension (OHTN) using analysis of covariance, adjusting for baseline values.

Abdominal obesity was defined as abdominal circumference $>102 \mathrm{~cm}$ in males and $>88 \mathrm{~cm}$ in females measured at the level of umbilicus. Pulse Pressure was defined as the difference between office SBP and DBP or between 24-H ambulatory SBP and DBP.

Comparisons between groups were made using twosample $t$-tests for continuous variables and Fisher's exact test, for categorical variables. Continuous variables are expressed as mean \pm standard deviation unless otherwise specified and between-group differences are expressed as mean and corresponding two-sided $95 \%$ confidence intervals. All analyses were performed using statistical analysis system (SAS) software version 9.4 (SAS Institute, Cary, NC, US). A $p$ value lower than 0.05 (two-sided) was considered significant. Reported $p$ values are based on nominal values not adjusted for multiple comparisons.

\section{Results}

\section{Population}

The distribution of the patient population in the RADIANCE-HTN SOLO trial was evenly matched between the RDN and Sham groups and has been previously reported [1]. The baseline characteristics of the perprotocol cohort treated with endovascular ultrasound RDN included in this analysis are summarized in (Supplemental Table S1).

\section{Univariate analysis}

All the variables assessed in the univariate model for the RDN group are listed in Table 1. The use of antihypertensive medications at screening, higher baseline dADBP, and OHTN were significant predictors, but not baseline dASBP; age, ethnicity, sex, estimated glomerular filtration rate (eGFR), hemoglobin A1C (HbA1C), body mass index (BMI), or the presence of sleep apnea. The presence of any untreated accessory artery was a negative predictor of the reduction in dASBP following RDN at 2 months. A similar univariate analysis was conducted in the Sham group and showed no variables as being significant predictors of BP response (Supplemental Table S2).

In responders to RDN (defined as reduction in dASBP $\geq$ $5 \mathrm{mmHg}$ ), the age and the presence of OHTN were positive predictors of response and the presence of any untreated accessories was a negative predictor of response. In the Sham group, there were no predictors of response identified (Table 2).

In super responders to RDN (defined as reduction in dASBP $\geq 15 \mathrm{mmHg}$ ), BMI, abdominal obesity, antihypertensive medications at screening, the presence of OHTN, baseline 24-H ambulatory heart rate (AHR), and the presence of side branches proximal to ablation, were significant predictors for BP-lowering effect of RDN. Whereas the presence of any untreated accessories was a negative predictor of response. There were no significant variables identified in the sham group (Supplemental Table S3).

\section{Multivariate and interaction analyses}

Results of the multivariate analysis are shown in Table 3. Baseline dADBP had a small but significant impact on response to RDN with respect to the fall in dASBP. Patients taking antihypertensive medication at screening who underwent a 12-week treatment interruption had a greater response to RDN at 2 months than those who were not medicated at screening. Of note baseline dASBP/dADBP in non-medicated $(N=11)$ and medicated patients $(N=53)$ were similar at $150 \pm 7.6 / 95 \pm 4.4 \mathrm{mmHg}$ and $151 \pm 7.3 / 93 \pm$ $4.4 \mathrm{mmHg}$, respectively. The presence of OHTN was also associated with a greater response to RDN albeit nonsignificant $(p=0.077)$.

A variety of interaction models were analyzed (Supplemental Tables S4 and S5) with the key finding from a 3-way interaction model that females with abdominal obesity had the greatest response to RDN in the per-protocol group (Table 4). There was no difference in the BP response to RDN between obese and non-obese males and gender alone had no impact on outcome and neither did age (data not shown). 
Table 1 Univariate analysis of predictors of response for change in daytime ambulatory systolic blood pressure from baseline to 2 months in the RDN group.

\begin{tabular}{|c|c|c|c|}
\hline Variable & Estimate parameter & Standard error & $p$ value \\
\hline Age & 0.1205 & 0.1206 & 0.3217 \\
\hline Male & 0.9242 & 2.4923 & 0.7120 \\
\hline Black Race & 1.1347 & 3.4717 & 0.7449 \\
\hline BMI & -0.2576 & 0.1994 & 0.2012 \\
\hline Abdominal obesity & -3.3343 & 2.4018 & 0.1701 \\
\hline Baseline eGFR & 0.0651 & 0.0834 & 0.4384 \\
\hline Sleep apnea & -3.9201 & 4.1141 & 0.3444 \\
\hline Hemoglobin A1c & 0.8367 & 1.9760 & 0.6734 \\
\hline Antihypertensive medications at screening (yes vs. no) & -6.4115 & 3.0965 & 0.0426 \\
\hline Baseline orthostatic hypertension & -6.9471 & 2.8698 & 0.0184 \\
\hline Baseline office systolic BP & 0.0413 & 0.0936 & 0.6610 \\
\hline Baseline office diastolic BP & -0.0368 & 0.1510 & 0.8084 \\
\hline Baseline daytime systolic ABP & -0.0848 & 0.1666 & 0.6124 \\
\hline Baseline daytime diastolic ABP & -0.6148 & 0.2649 & 0.0236 \\
\hline Baseline nighttime systolic ABP & 0.0117 & 0.0992 & 0.9068 \\
\hline Baseline nighttime diastolic ABP & -0.0340 & 0.1515 & 0.8230 \\
\hline 24-H systolic ABP & 0.0183 & 0.1542 & 0.9058 \\
\hline 24-H diastolic ABP & -0.2926 & 0.2558 & 0.2570 \\
\hline Baseline office pulse pressure & 0.0800 & 0.1122 & 0.4786 \\
\hline Baseline $24-\mathrm{H}$ ambulatory pulse pressure & 0.1511 & 0.1702 & 0.3781 \\
\hline Baseline office heart rate & -0.0570 & 0.0969 & 0.5587 \\
\hline Baseline $24-\mathrm{H}$ ambulatory heart rate & -0.1438 & 0.1202 & 0.2361 \\
\hline Total number of ablations & -0.7343 & 1.3117 & 0.5777 \\
\hline Average main artery diameter left & 0.8442 & 1.5629 & 0.5910 \\
\hline Average main artery diameter right & -0.5303 & 1.7371 & 0.7612 \\
\hline Vessel length (left renal) & 0.0086 & 0.0986 & 0.9309 \\
\hline Vessel length (right renal) & -0.0122 & 0.1021 & 0.9055 \\
\hline Presence of any untreated accessory & 5.4188 & 2.7034 & 0.0494 \\
\hline Presence of side branches proximal to ablations & -4.3473 & 3.4306 & 0.2098 \\
\hline $\begin{array}{l}\text { Farthest distance bilaterally from distal ablation to } \\
\text { parenchyma }\end{array}$ & 0.0049 & 0.1290 & 0.9698 \\
\hline Operator case number & 0.2189 & 0.8714 & 0.8025 \\
\hline Contrast volume used & -0.0149 & 0.0179 & 0.4087 \\
\hline Duration of procedure & -0.0262 & 0.0541 & 0.6296 \\
\hline Fluoro time & 0.0799 & 0.2078 & 0.7019 \\
\hline Paradise catheter time & 0.0967 & 0.0681 & 0.1604 \\
\hline Number of different balloon sizes used in a patient & -1.7074 & 1.6204 & 0.2961 \\
\hline
\end{tabular}

$\overline{A B P}$ ambulatory blood pressure, $B M I$ body mass index, $B P$ blood pressure, $e G F R$ estimated glomerular filtration rate.

Statistically significant $p$-values are in bold.

\section{Patients with orthostatic hypertension}

The results from the multivariate analysis suggested an influence of the presence of OHTN on ABP response to RDN in the per-protocol population. The baseline characteristics of the per-protocol RDN group were similar between patients with and without OHTN with the exception of less Caucasian ethnicity and higher baseline eGFR in patients with OHTN (Supplemental Table S6).
The presence of OHTN at baseline (systolic or diastolic OHTN) was associated with a larger reduction in dASBP and dADBP 2 months after RDN (Table 5). Figure 1 shows that $92 \%(12 / 13)$ patients with OHTN demonstrated a reduction in dASBP greater than $5 \mathrm{mmHg}$, whereas $63 \%$ (32/51) patients without OHTN had such a reduction and even $22 \%(11 / 51)$ had a rise in dASBP. There was no influence of OHTN on the change in nighttime BP, 24-H BP, 24-H AHR or office BP or HR. Further analysis 
Table 2 Characteristics of responders (dASBP drop $\geq 5 \mathrm{mmHg}$ ).

\begin{tabular}{|c|c|c|c|c|c|c|}
\hline \multirow[b]{2}{*}{ Characteristics } & \multicolumn{3}{|c|}{ Renal denervation } & \multicolumn{3}{|l|}{ Sham procedure } \\
\hline & $\begin{array}{l}\text { Responder } \\
(N=44)\end{array}$ & $\begin{array}{l}\text { Non-Responder } \\
(N=20)\end{array}$ & $p$ value & $\begin{array}{l}\text { Responder } \\
(N=15)\end{array}$ & $\begin{array}{l}\text { Non-Responder } \\
(N=43)\end{array}$ & $p$ value \\
\hline Age (years) & $52.3 \pm 10.0$ & $58.7 \pm 8.7$ & 0.017 & $51.1 \pm 9.7$ & $55.0 \pm 10.2$ & 0.202 \\
\hline Male & $68.2 \%(30 / 44)$ & $50.0 \%(10 / 20)$ & 0.164 & $60.0 \%(9 / 15)$ & $62.8 \%(27 / 43)$ & 0.848 \\
\hline Black Race & $13.6 \%(6 / 44)$ & $15.0 \%(3 / 20)$ & 0.884 & $20.0 \%(3 / 15)$ & $16.3 \%(7 / 43)$ & 0.743 \\
\hline BMI $\left(\mathrm{kg} / \mathrm{m}^{2}\right)$ & $30.0 \pm 5.8$ & $29.5 \pm 6.7$ & 0.733 & $29.8 \pm 6.8$ & $29.7 \pm 4.4$ & 0.953 \\
\hline Abdominal obesity ${ }^{\mathrm{a}}$ & $59.1 \%(26 / 44)$ & $47.4 \%(9 / 19)$ & 0.390 & $53.3 \%(8 / 15)$ & $69.8 \%(30 / 43)$ & 0.249 \\
\hline Baseline eGFR $\left(\mathrm{ml} / \mathrm{min} / 1.73 \mathrm{~m}^{2}\right)^{\mathrm{a}}$ & $82.2 \pm 13.3$ & $86.4 \pm 16.9$ & 0.288 & $86.3 \pm 17.4$ & $82.1 \pm 13.7$ & 0.343 \\
\hline Sleep apnea & $11.4 \%(5 / 44)$ & $5.0 \%(1 / 20)$ & 0.418 & $13.3 \%(2 / 15)$ & $11.6 \%(5 / 43)$ & 0.861 \\
\hline Hemoglobin A1c $(\%)^{\mathrm{a}}$ & $5.4 \pm 0.7$ & $5.5 \pm 0.4$ & 0.641 & $5.6 \pm 0.9$ & $5.5 \pm 0.5$ & 0.699 \\
\hline Antihypertensive Medications at Screening (yes vs. no) & $86.4 \%(38 / 44)$ & $75.0 \%(15 / 20)$ & 0.264 & $66.7 \%(10 / 15)$ & $79.1 \%(34 / 43)$ & 0.334 \\
\hline Baseline orthostatic hypertension & $27.3 \%(12 / 44)$ & $5.0 \%(1 / 20)$ & 0.040 & $20.0 \%(3 / 15)$ & $16.3 \%(7 / 43)$ & 0.743 \\
\hline Baseline office systolic BP (mmHg) & $153.6 \pm 11.9$ & $157.0 \pm 15.1$ & 0.341 & $146.0 \pm 14.1$ & $154.6 \pm 15.8$ & 0.068 \\
\hline Baseline office diastolic $\mathrm{BP}(\mathrm{mmHg})$ & $99.8 \pm 7.2$ & $100.3 \pm 9.8$ & 0.827 & $94.2 \pm 8.5$ & $99.6 \pm 9.8$ & 0.062 \\
\hline Baseline daytime systolic $\mathrm{ABP}(\mathrm{mmHg})$ & $150.5 \pm 7.7$ & $150.5 \pm 6.5$ & 0.995 & $148.0 \pm 8.6$ & $150.1 \pm 10.3$ & 0.472 \\
\hline Baseline daytime diastolic $\mathrm{ABP}(\mathrm{mmHg})$ & $93.9 \pm 4.7$ & $92.5 \pm 3.5$ & 0.262 & $93.0 \pm 4.8$ & $93.3 \pm 5.6$ & 0.855 \\
\hline Baseline nighttime systolic ABP $(\mathrm{mmHg})^{\mathrm{a}}$ & $130.5 \pm 12.7$ & $130.9 \pm 11.7$ & 0.909 & $130.8 \pm 15.6$ & $131.7 \pm 13.2$ & 0.828 \\
\hline Baseline nighttime diastolic ABP $(\mathrm{mmHg})^{\mathrm{a}}$ & $78.7 \pm 8.4$ & $78.4 \pm 7.4$ & 0.918 & $80.0 \pm 7.8$ & $80.0 \pm 7.4$ & 0.997 \\
\hline 24-H systolic ABP (mmHg) & $142.5 \pm 8.4$ & $143.6 \pm 6.9$ & 0.638 & $141.7 \pm 10.3$ & $143.7 \pm 10.8$ & 0.534 \\
\hline 24-H diastolic ABP (mmHg) & $87.9 \pm 5.1$ & $87.4 \pm 3.9$ & 0.691 & $88.2 \pm 4.7$ & $88.6 \pm 5.6$ & 0.807 \\
\hline Baseline office pulse pressure (mmHg) & $53.8 \pm 10.0$ & $56.7 \pm 12.4$ & 0.327 & $51.8 \pm 10.0$ & $55.0 \pm 12.4$ & 0.374 \\
\hline Baseline $24-\mathrm{H}$ ambulatory pulse pressure $(\mathrm{mmHg})$ & $54.6 \pm 7.3$ & $56.1 \pm 6.7$ & 0.432 & $53.5 \pm 9.3$ & $55.1 \pm 9.2$ & 0.565 \\
\hline Baseline office heart rate (bpm) & $71.8 \pm 11.9$ & $72.9 \pm 14.2$ & 0.757 & $68.1 \pm 8.8$ & $71.8 \pm 12.9$ & 0.315 \\
\hline Baseline $24-\mathrm{H}$ ambulatory heart rate (bpm) & $72.6 \pm 7.7$ & $73.1 \pm 14.1$ & 0.871 & $71.5 \pm 10.2$ & $70.5 \pm 9.8$ & 0.725 \\
\hline Total number of ablations & $5.5 \pm 1.0$ & $5.5 \pm 0.8$ & 0.773 & & & \\
\hline Average main artery diameter left $(\mathrm{mm})$ & $5.5 \pm 0.7$ & $5.4 \pm 0.9$ & 0.623 & & & \\
\hline Average main artery diameter right (mm) & $5.4 \pm 0.7$ & $5.0 \pm 0.7$ & 0.053 & & & \\
\hline Vessel length (left renal) (mm) & $38.0 \pm 12.8$ & $38.8 \pm 11.6$ & 0.807 & & & \\
\hline Vessel length (right renal) (mm) & $43.7 \pm 11.7$ & $47.0 \pm 12.4$ & 0.317 & & & \\
\hline Presence of any untreated accessory & $15.9 \%(7 / 44)$ & $45.0 \%(9 / 20)$ & 0.013 & & & \\
\hline Presence of side branches proximal to ablations & $15.9 \%(7 / 44)$ & $10.0 \%(2 / 20)$ & 0.528 & & & \\
\hline $\begin{array}{l}\text { Farthest distance bilaterally from distal ablation to } \\
\text { parenchyma }\end{array}$ & $19.2 \pm 9.9$ & $17.4 \pm 8$ & 0.487 & & & \\
\hline Operator case number & $1.9 \pm 1.5$ & $2.3 \pm 1.3$ & 0.332 & & & \\
\hline Contrast volume used $(\mathrm{cc})^{\mathrm{a}}$ & $145.5 \pm 64.3$ & $119.8 \pm 72.6$ & 0.168 & & & \\
\hline Duration of procedure (min) & $72.0 \pm 21.1$ & $65.9 \pm 25.3$ & 0.320 & & & \\
\hline Fluoro time $(\min )^{\mathrm{a}}$ & $13.0 \pm 4.3$ & $14.1 \pm 8.8$ & 0.589 & & & \\
\hline Paradise catheter time (min) & $32.9 \pm 18.1$ & $33.0 \pm 17.0$ & 0.985 & & & \\
\hline Number of different balloon sizes used in patient & $1.8 \pm 0.8$ & $1.5 \pm 0.6$ & 0.114 & & & \\
\hline
\end{tabular}

Data displayed as $\%(n / N)$ and mean \pm standard deviation.

$A B P$ ambulatory blood pressure, $B M I$ body mass index, $B P$ blood pressure, $e G F R$ estimated glomerular filtration rate.

${ }^{a}$ Contrast volume was missing from one patient and flouro time was missing from three patients in the renal denervation responder group. Abdominal obesity status and contrast volume each had one patient with missing data in the renal denervation non-responder group. eGFR, Nighttime SBP and Nighttime DBP each had one patient with missing data and hemoglobin A1C had two patients with missing data in the sham non-responder group.

Statistically significant $p$-values are in bold. 
Table 3 Multivariate analysis of predictors of response for change in daytime ambulatory systolic blood pressure from baseline to 2 months in the RDN group.

\begin{tabular}{llll}
\hline Variable & $\begin{array}{l}\text { Estimate } \\
\text { parameter }\end{array}$ & Standard error & $p$ value \\
\hline $\begin{array}{l}\text { Antihypertensive medications } \\
\text { at screening (yes vs. no) }\end{array}$ & -6.4952 & 2.9525 & 0.0317 \\
$\begin{array}{l}\text { Baseline orthostatic } \\
\text { hypertension }\end{array}$ & -5.0303 & 2.7955 & 0.0770 \\
$\begin{array}{l}\text { Baseline daytime diastolic ABP } \\
\text { B }\end{array}$ & -0.5953 & 0.2566 & 0.0238 \\
\hline
\end{tabular}

$A B P$ ambulatory blood pressure.

Abdominal obesity, antihypertensive medications at screening (yes vs. no), baseline orthostatic hypertension, baseline daytime diastolic ABP, presence of any untreated accessory, and paradise catheter time were the variables included in the multivariate regression model. Then backward selection with a stay criterion of 0.20 was used to select predictors.

Table 4 Three-way interaction model of treatment arm, sex, and abdominal obesity on change in daytime ambulatory blood pressure from baseline to 2 months.

\begin{tabular}{|c|c|c|c|c|c|}
\hline \multicolumn{5}{|l|}{ Variable } & $p$ value \\
\hline \multicolumn{5}{|l|}{ Treatment arm } & $<0.001$ \\
\hline \multicolumn{5}{|l|}{ Sex } & 0.967 \\
\hline \multicolumn{5}{|l|}{ Treatment arm $\times \operatorname{sex}$} & 0.879 \\
\hline \multicolumn{5}{|l|}{ Abdominal obesity } & 0.811 \\
\hline \multicolumn{5}{|c|}{ Treatment arm $\times$ abdominal obesity } & 0.006 \\
\hline \multicolumn{5}{|c|}{ Treatment arm $\times$ abdominal obesity } & 0.913 \\
\hline \multicolumn{5}{|c|}{ Treatment arm $\times \operatorname{sex} \times$ abdominal obesity } & 0.021 \\
\hline Treatment Arm & Sex & $\begin{array}{l}\text { Abdominal } \\
\text { Obesity }\end{array}$ & $n$ & $\begin{array}{l}\text { Change in } \\
\mathrm{SBP} \text { at } 2 \mathrm{M} \\
(\mathrm{mmHg})(\mathrm{le} \\
\text { squares me }\end{array}$ & $\begin{array}{l}\text { daytime } \\
\text { I } \\
\text { east } \\
\text { an) }\end{array}$ \\
\hline Renal denervation & Female & Yes & 17 & -12.301 & \\
\hline Renal denervation & Female & No & 6 & -3.064 & \\
\hline Renal denervation & Male & Yes & 18 & -7.990 & \\
\hline Renal denervation & Male & No & 22 & -7.780 & \\
\hline Sham procedure & Female & Yes & 17 & 3.402 & \\
\hline Sham procedure & Female & No & 5 & -6.315 & \\
\hline Sham procedure & Male & Yes & 21 & -0.347 & \\
\hline Sham procedure & Male & No & 15 & -1.846 & \\
\hline
\end{tabular}

demonstrated that neither baseline office standing SBP or DBP nor the difference between baseline office standing and seated SBP or DBP were significant predictors of change in ambulatory or office BP and HR (data not shown). In the RDN patients with OHTN at baseline, 69\% (9/13) no longer met the definition of OHTN at 2 months after RDN.

\section{Discussion}

Previous studies have identified baseline SBP, younger age, higher eGFR, higher HR, use of central sympatholytic agents or aldosterone antagonists, indices of baseline sympathetic overdrive, and various biological markers as markers of response to catheter-based RF RDN [10, 16-22].

In addition to being limited to populations treated with $\mathrm{RF}$ energy to achieve renal sympathectomy, these studies mostly included patients taking antihypertensive medications. Given that dynamic patterns of adherence and non-adherence to antihypertensive drugs in trials are now established, it might be expected that this would have confounding effects on clinical outcomes [23]. The RADIANCE-HTN SOLO study thus provides us a unique opportunity to evaluate predictors of BP-lowering response to endovascular ultrasound-based RDN in a non-medicated population, eliminating thus the confounding effect of medication use.

In this post-hoc analysis of the RADIANCE-HTN SOLO trial, we found that the predictors of a larger response to RDN were mainly linked to heightened sympathetic nervous system (SNS) activity in addition to anatomical predictors. Indeed, the positive predictors of BP response to endovascular ultrasound RDN were use of antihypertensive medications at screening, higher baseline dADBP, abdominal obesity, female sex, and OHTN. Patients receiving antihypertensive medications at screening may have demonstrated an enhanced response to RDN due to medicationinduced priming of the SNS at the time of screening even though baseline BPs were similar between the groups [24]. Patients with higher baseline dADBP having greater response suggests that in middle-aged patients with milder forms of combined hypertension, there may be heightened SNS activity and that increased DBP, reflecting increased systemic vascular resistance which is under neurohumoral control, might act as a surrogate marker for this [25]. Indeed, it has been recognized that whole-body sympathetic neural activity rises in both males and females after the age of 30 years [26]. Notably, and in contrast to earlier studies in patients with resistant hypertension, baseline office SBP and dASBP did not influence the response to RDN and this was also observed in the SPYRAL HTN-OFF MED study, again possibly related to the selection of patients with milder forms of hypertension in both the SPYRAL HTN-OFF MED and RADIANCE-HTN SOLO studies [1, 4]. Also, increased DBP is associated with increased systemic vascular resistance, which is more easily reversible by any antihypertensive treatment, whereas increased SBP is associated with increased aortic stiffness and structural arterial changes which are much less reversible [27].

Finally, muscle sympathetic nerve activity (MSNA) is an established surrogate marker for SNS activity. In males and 
Table 5 Ambulatory BP and HR changes from baseline to 2 months for RDN patients with and without orthostatic hypertension.

\begin{tabular}{lcll}
\hline Parameters & $\begin{array}{l}\text { With orthostatic hypertension } \\
(N=13)\end{array}$ & $\begin{array}{l}\text { Without orthostatic hypertension } \\
(N=51)\end{array}$ & $p$ value \\
\hline Change from baseline to 2 & months & & \\
Daytime SBP (mmHg) & $-14.00 \pm 6.26$ & $-7.05 \pm 9.82$ & 0.022 \\
Daytime DBP (mmHg) & $-8.92 \pm 5.10$ & $-4.47 \pm 5.88$ & 0.025 \\
Nighttime SBP (mmHg) & $-7.07 \pm 11.75$ & $-4.24 \pm 12.32$ & 0.641 \\
Nighttime DBP (mmHg) & $-5.28 \pm 8.18$ & $-3.13 \pm 9.00$ & 0.488 \\
24-H SBP (mmHg) & $-10.98 \pm 7.68$ & $-6.01 \pm 9.04$ & 0.094 \\
24-H DBP (mmHg) & $-7.28 \pm 5.55$ & $-3.93 \pm 6.01$ & 0.111 \\
24-H HR (bpm) & $0.55 \pm 4.52$ & $1.24 \pm 4.68$ & 0.700 \\
Office SBP (mmHg) & $-12.62 \pm 13.77$ & $-9.27 \pm 12.57$ & 0.520 \\
Office DBP (mmHg) & $-3.08 \pm 10.56$ & $-5.63 \pm 7.92$ & 0.425 \\
Office HR (bpm) & $-0.31 \pm 8.83$ & $-0.82 \pm 10.19$ & 0.761 \\
\hline
\end{tabular}

Data displayed as mean \pm standard deviation.

$D B P$ diastolic blood pressure, $H R$ heart rate, $S B P$ systolic blood pressure.

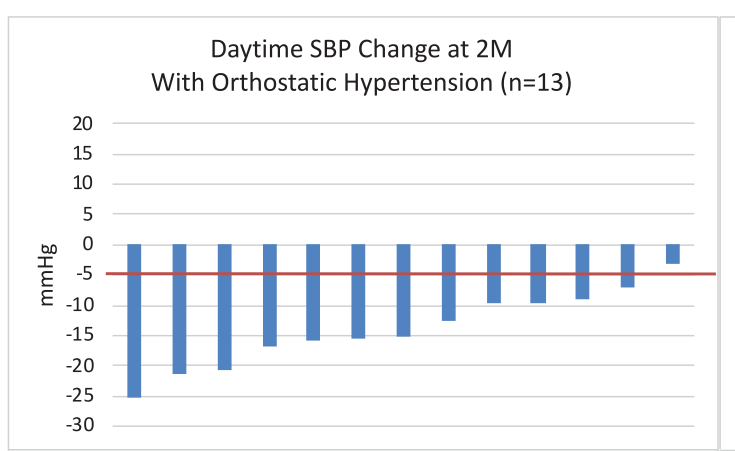

Nighttime SBP Change at $2 \mathrm{M}$ With Orthostatic Hypertension $(n=13)$

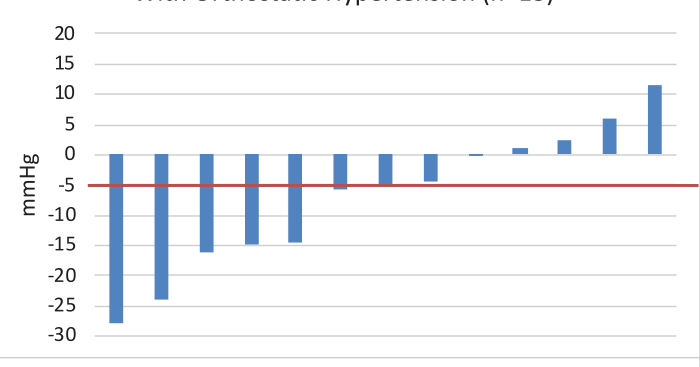

24h SBP Change at 2M with Orthostatic Hypertension ( $n=13$ )

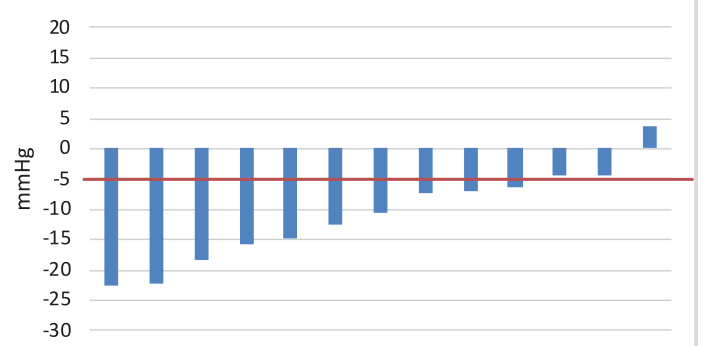

Daytime SBP Change at 2M Without Orthostatic Hypertension $(n=51)$

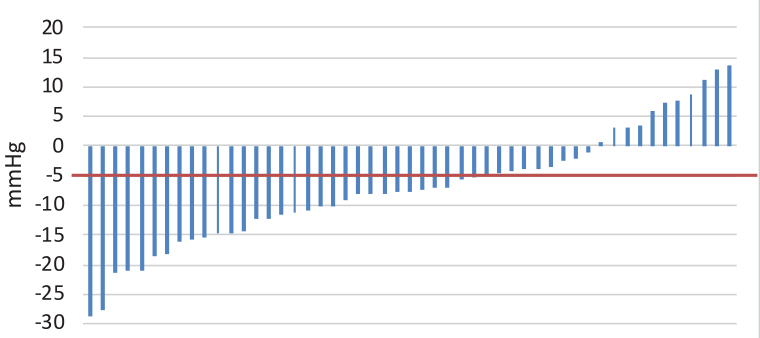

Nighttime SBP Change at $2 \mathrm{M}$ Without Orthostatic Hypertension ( $n=51$ )

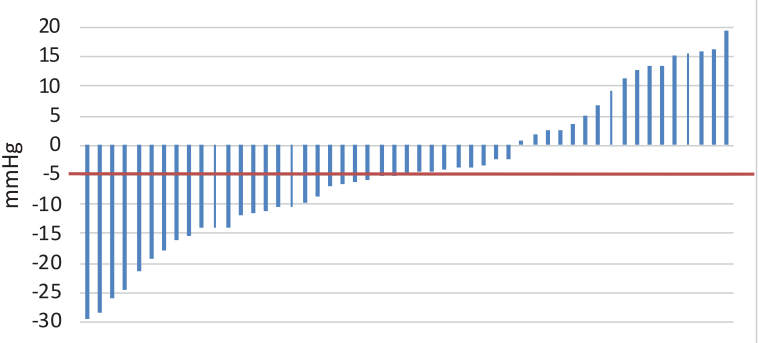

24h SBP Change at $2 \mathrm{M}$ Without Orthostatic Hypertension $(n=51)$

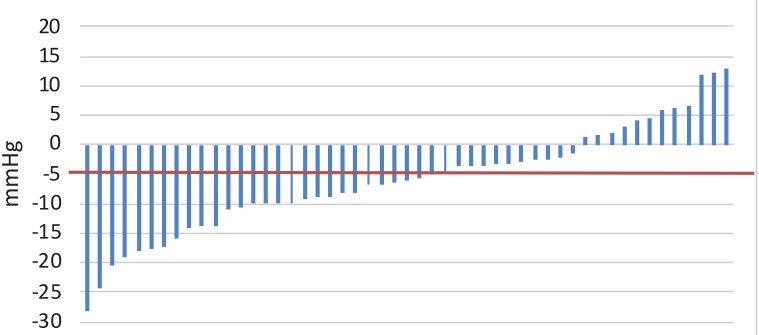

Fig. 1 Change in SBP following RDN in individual patients with and without orthostatic hypertension. Data shown for patients with orthostatic hypertension (left panels) and without orthostatic hypertension (right panels) for daytime SBP (top panels), nighttime SBP (middle panels), and 24-H SBP (bottom panels). 
females more than 40 years of age, MSNA correlates significantly with mean arterial pressure, which has a major contribution from diastolic pressure compared to systolic pressure [28]. It is also known that early HTN is characterized by increased cardiac output and established HTN is characterized by high vascular resistance [29]. Hence diastolic BP and vascular resistance are better surrogates of SNS overactivity and may therefore be better predictors of response to RDN compared to systolic BP. These findings are consistent with SPYRAL HTN-OFF MED study findings.

In the three-way interaction model, females with abdominal obesity showed striking reduction in BP following RDN. Abdominal obesity is also a known feature of the metabolic syndrome characterized by higher baseline SNS activity [30]. Moreover, the mean age of the females in our study was 54 years and increasing age in women has been demonstrated to result in striking upregulation in MSNA independent of the menopause as well as increase in BP [28]. Thus, obese females may represent a group for whom strategies targeting sympathetic regulation of the circulation may be particularly effective [31].

The presence of systolic and/or diastolic OHTN was a positive predictor of dASBP and dADBP lowering following RDN in the univariate analysis although this did not reach significance in the multivariate analysis. Interestingly, the OHTN phenotype was also associated with younger age, female sex, higher BMI, and eGFR, but the sample size may have been too small to achieve statistical significance in most factors (Supplemental Table S6).

A preliminary report from the SPYRAL HTN-OFF MED study noted OHTN as a predictor of response to RF-based RDN and notably, in both this study and herein, patients with OHTN exhibited larger BP reduction following RDN with markedly reduced variability of dASBP response compared to the overall per-protocol treatment groups [32]. Of interest, in RADIANCE-HTN SOLO only daytime BP parameters were significantly reduced in patients with OHTN with no effects on nocturnal and 24-h BP levels or HR perhaps due to reduction in SNS activation with recumbency and sleep and resultant lessened impact of sympathomodulatory treatment.

The pathophysiology of OHTN is poorly understood but it is considered a manifestation of SNS dysfunction [33]. Systolic OHTN is associated with older age and a stiff circulation, whereas diastolic OHTN (seen in the majority of our patients) is associated with higher resting cardiac output, HR and urinary norepinephrine output suggesting increased baseline SNS activity [34]. It is associated with increased activity of the SNS to the resistance vessels causing vasoconstriction and in particular increase in diastolic pressure. Notably, it was previously shown that BP lowering associated with RDN was associated with a significant reduction in total peripheral resistance [35].
Together these factors strongly suggest that in hypertensive patients with OHTN, a treatment such as RDN that reduces SNS activity might be effective and that OHTN per se may be valuable as a screening tool for neurogenic hypertension and improved response to RDN [36].

\section{Conclusions}

In this study, the positive predictors of response to RDN were higher baseline dADBP and the use of antihypertensive medications at screening. The presence of untreated accessory arteries was a negative predictor of response to RDN in the univariate analysis without reaching significance in the multivariate analysis. The presence of OHTN did not reach significance in the multivariate analysis but is an interesting finding and needs to be explored in future studies with bigger numbers. Obese females also appeared to be better responders to RDN in the interaction model. All of the findings noted above merit exploration in larger randomized controlled trials as identifying patients most suitable for RDN remains a clinical priority given that efficacy and safety of this therapy has been established [1-4].

\section{Limitations of this study}

This study was a post-hoc analysis of a small sample size albeit from a rigorously conducted randomized clinical trial with objective endpoints and negligible data loss. In as such, our results can only be considered as hypothesis generating. We focused on the per-protocol population to ensure minimization of confounding through contaminating effects of medication. However, we did not strictly test blood or urine for drug metabolites so unauthorized consumption of antihypertensive medications cannot be completely ruled out. On the other hand, since these findings occurred in the setting of a well-controlled trial in patients off antihypertensive medications, they may be less applicable in real-world clinical practice. Finally, OHTN is ideally recorded as the difference between lying and standing BPs rather than seated and standing. If anything, we are likely to have diagnosed less patients with OHTN as a result although every single patient that demonstrated OHTN in our study showed BP reduction with RDN.

\section{Summary}

\section{What is known about this topic}

- RADIANCE-HTN SOLO study results have shown significant BP reduction with endovascular ultrasound RDN albeit with variable response. 
- Previous RDN studies using RF energy have looked at predictors of response in patients on BP-lowering medication.

- Predictors of response can vary with energy modality used (RF vs. ultrasound), study design, and patient population studied.

\section{What this study adds}

- This is the first evaluation of clinical, anatomical, and procedural predictors of response specific to endovascular ultrasound RDN.

- The study design offers a unique opportunity to evaluate predictors in absence of BP-lowering drugs in the study cohort.

- The study has identified novel predictors (abdominal obesity, orthostatic hypertension) that show bigger BP response with RDN and should be evaluated in future studies.

Acknowledgements Andrea Scicli, Ph.D., an employee of ReCor Medical, helped with editing and formatting of this article. She was compensated by ReCor Medical for her work.

Funding The RADIANCE-HTN SOLO study was funded by ReCor Medical (Palo Alto, CA, USA).

\section{Compliance with ethical standards}

Conflict of interest MS has received grant support and personal fees from ReCor Medical. RES has received grant support and personal fees from ReCor Medical, Medtronic, and Ablative Solutions. AJK reports Institutional funding to Columbia University and/or Cardiovascular Research Foundation from Medtronic, Boston Scientific, Abbott Vascular, Abiomed, CSI, CathWorks, Siemens, Philips, ReCor Medical. In addition to research grants, institutional funding includes fees paid to Columbia University and/or Cardiovascular Research Foundation for speaking engagements and/or consulting. Personal: Consulting: Neurotronic; Travel Expenses/Meals from Medtronic, Boston Scientific, Abbott Vascular, Abiomed, CSI, CathWorks, Siemens, Philips, ReCor Medical, Chiesi, OpSens, Zoll, and Regeneron. FM is supported by Deutsche Gesellschaft für Kardiologie (DGK), and Deutsche Forschungsgemeinschaft (SFB TRR219) and has received scientific support and speaker honoraria from Bayer, Boehringer Ingelheim, Medtronic and ReCor Medical. JD has received grant support from ReCor Medical, Medtronic, Boston Scientific, Abbott Vascular, Acist Medical, Astra Zeneca, Pie Medical, and Pulse Cath and has received personal fees from ReCor Medical, Medtronic, Acist Medical, Boston Scientific, Pie Medical, and Pulse Cath. JB has received grant support from ReCor Medical and Ablative Solutions. PL has received grant support and personal fees from ReCor Medical, Edwards, and Abbott and personal fees from Medtronic and Occlutech. PG has received grant support from Recor and Ablative Solutions. KS has received grant support and personal fees from ReCor Medical and Medtronic and grant support from CSI. NDLF has received grant support and personal fees from ReCor Medical. LCR has received personal fees and other support from ReCor Medical. AP has received personal fees from ReCor Medical, Medtronic, CVRx, Ablative Solutions, Dynamics, ROX Medical and grants from Medtronic, Ablative Solutions, ReCor Medical and CVRx. PJB reports institutional funding by the European Commission, Recor, Medtronic, Ablative Solutions, Fresenius and BBraun. MAW has received personal fees from ReCor Medical, Medtronic, Boston Scientific, and Ablative Solutions. ASPS has received personal fees from ReCor Medical, Medtronic and Philips. MJB has received personal fees from ReCor Medical and Medtronic. NCB is an employee of ReCor Medical and has multiple patents in renal denervation issued. LC is an employee of ReCor Medical. MA has received research grants from The French Ministry of Health, Quantum genomics and European H2020 program; has received grant support and non-financial support from ReCor Medical and Idorsia; and has received personal fees from CVRx. MDL has received personal fees from ReCor Medical, Medtronic, CVRx, Ablative Solutions, Vascular Dynamics, ROX Medical and Tarilan Laser Technologies and grants from Medtronic. All other authors declare no competing interests.

Publisher's note Springer Nature remains neutral with regard to jurisdictional claims in published maps and institutional affiliations.

Open Access This article is licensed under a Creative Commons Attribution 4.0 International License, which permits use, sharing, adaptation, distribution and reproduction in any medium or format, as long as you give appropriate credit to the original author(s) and the source, provide a link to the Creative Commons license, and indicate if changes were made. The images or other third party material in this article are included in the article's Creative Commons license, unless indicated otherwise in a credit line to the material. If material is not included in the article's Creative Commons license and your intended use is not permitted by statutory regulation or exceeds the permitted use, you will need to obtain permission directly from the copyright holder. To view a copy of this license, visit http://creativecommons. org/licenses/by/4.0/.

\section{References}

1. Azizi M, Schmieder RE, Mahfoud F, Weber MA, Daemen J, Davies J, et al. Endovascular ultrasound renal denervation to treat hypertension (RADIANCE-HTN SOLO): a multicentre, international, single-blind, randomised, sham-controlled trial. Lancet. 2018;391:2335-45.

2. Townsend RR, Mahfoud F, Kandzari DE, Kario K, Pocock S, Weber MA, et al. Catheter-based renal denervation in patients with uncontrolled hypertension in the absence of antihypertensive medications (SPYRAL HTN-OFF MED): a randomised, shamcontrolled, proof-of-concept trial. Lancet. 2017;390:2160-70.

3. Kandzari DE, Böhm M, Mahfoud F, Townsend RR, Weber MA, Pocock S, et al. Effect of renal denervation on blood pressure in the presence of antihypertensive drugs: 6-month efficacy and safety results from the SPYRAL HTN-ON MED proof-of-concept randomised trial. Lancet. 2018;391:2346-55.

4. Böhm M, Kario K, Kandzari DE, Mahfoud F, Weber MA, Schmieder RE, et al. Efficacy of catheter-based renal denervation in the absence of antihypertensive medications (SPYRAL HTNOFF MED Pivotal): a multicentre, randomised, sham-controlled trial. Lancet. 2020;395:1444-51.

5. Azizi M, Schmieder RE, Mahfoud F, Weber MA, Daemen J, Lobo MD, et al. Six-Month results of treatment-blinded medication titration for hypertension control after randomization to endovascular ultrasound renal denervation or a sham procedure in the RADIANCE-HTN SOLO trial. Circulation. 2019;139: 2542-53. 
6. Weber MA, Mahfoud F, Schmieder RE, Kandzari DE, Tsioufis $\mathrm{KP}$, Townsend RR, et al. Renal denervation for treating hypertension: current scientific and clinical evidence. JACC Cardiovasc Interv. 2019;12:1095-105.

7. Mahfoud F, Azizi M, Ewen S, Pathak A, Ukena C, Blankestijn PJ, et al. Proceedings from the 3rd European Clinical Consensus Conference for clinical trials in device-based hypertension therapies. Eur Heart J. 2020;41:1588-99.

8. Courand P-Y, Pereira H, Del Giudice C, Gosse P, Monge M, Bobrie $G$, et al. Abdominal aortic calcifications influences the systemic and renal hemodynamic response to renal denervation in the DENERHTN (Renal Denervation for Hypertension) trial. J Am Heart Assoc. 2017;6:1-16.

9. Fengler K, Rommel K-P, Blazek S, von Roeder M, Besler C, Hartung P, et al. Predictors for profound blood pressure response in patients undergoing renal sympathetic denervation. J Hypertens. 2018;36:1578-84.

10. Gosse P, Cremer A, Pereira H, Bobrie G, Chatellier G, Chamontin $\mathrm{B}$, et al. Twenty-four-hour blood pressure monitoring to predict and assess impact of renal denervation. Hypertension. 2017;69:494-500.

11. Tadic M, Cuspidi C, Grassi G. Heart rate as a predictor of cardiovascular risk. Eur J Clin Investig. 2018;48. https://doi.org/10. 1111/eci.12892.

12. Vriz O, Soon G, Lu H, Weder AB, Canali C, Palatini P. Does orthostatic testing have any role in the evaluation of the young subject with mild hypertension?: An insight from the HARVEST study. Am J Hypertens. 1997;10:546-51.

13. Magkas N, Tsioufis C, Thomopoulos C, Dilaveris P, Georgiopoulos $\mathrm{G}$, Doumas $\mathrm{M}$, et al. Orthostatic hypertension: from pathophysiology to clinical applications and therapeutic considerations. J Clin Hypertens. 2019;21:426-33.

14. Rea RF, Hamdan M. Baroreflex control of muscle sympathetic nerve activity in borderline hypertension. Circulation 1990. https://doi.org/10.1161/01.CIR.82.3.856.

15. Shibao C, Gamboa A, Diedrich A, Ertl AC, Chen KY, Byrne DW, et al. Autonomic contribution to blood pressure and metabolism in obesity. Hypertension. 2007;49:27-33.

16. Kandzari DE, Bhatt DL, Brar S, Devireddy CM, Esler M, Fahy M, et al. Predictors of blood pressure response in the SYMPLICITY HTN-3 trial. Eur Heart J. 2015;36:219-27.

17. Krum H, Schlaich M, Whitbourn R, Sobotka PA, Sadowski J, Bartus $\mathrm{K}$, et al. Catheter-based renal sympathetic denervation for resistant hypertension: a multicentre safety and proof-of-principle cohort study. Lancet. 2009;373:1275-81.

18. Rohla M, Nahler A, Lambert T, Reiter C, Gammer V, Grund M, et al. Predictors of response to renal denervation for resistant arterial hypertension: a single center experience. J Hypertens. 2016;34:123-9.

19. Persu A, Azizi M, Jin Y, Volz S, Rosa J, Fadl Elmula FEM, et al. Hyperresponders vs. nonresponder patients after renal denervation: do they differ? J Hypertens. 2014;32:2422-7.

20. Symplicity HTN-2 Investigators, Esler MD, Krum H, et al. RE et al. Renal sympathetic denervation in patients with treatmentresistant hypertension (The Symplicity HTN-2 Trial): a randomised controlled trial. Lancet. 2010;376:1903-9.

21. Chinushi M, Izumi D, Iijima K, Suzuki K, Furushima H, Saitoh O, et al. Blood pressure and autonomic responses to electrical stimulation of the renal arterial nerves before and after ablation of the renal artery. Hypertension. 2013;61:450-6.

22. Dörr O, Liebetrau C, Möllmann H, Gaede L, Troidl C, Rixe J, et al. Soluble fms-like tyrosine kinase-1 and endothelial adhesion molecules (intercellular cell adhesion molecule-1 and vascular cell adhesion molecule-1) as predictive markers for blood pressure reduction after renal sympathetic denervation. Hypertension. 2014;63:984-90.

23. Azizi M, Pereira H, Hamdidouche I, Gosse P, Monge M, Bobrie $\mathrm{G}$, et al. Adherence to antihypertensive treatment and the blood pressure-lowering effects of renal denervation in the renal denervation for hypertension (DENERHTN) trial. Circulation. 2016;134:847-57.

24. Del Colle S, Morello F, Rabbia F, Milan A, Naso D, Puglisi E, et al. Antihypertensive drugs and the sympathetic nervous system. J Cardiovasc Pharmacol. 2007;50:487-96.

25. Guyenet PG. The sympathetic control of blood pressure. Nat Rev Neurosci. 2006;7:335-46.

26. Joyner MJ, Charkoudian N, Wallin BG. Sympathetic nervous system and blood pressure in humans: individualized patterns of regulation and their implications. Hypertension. 2010;56:10-6.

27. Mahfoud F, Bakris G, Bhatt DL, Esler M, Ewen S, Fahy M, et al. Reduced blood pressure-lowering effect of catheter-based renal denervation in patients with isolated systolic hypertension: data from SYMPLICITY HTN-3 and the Global SYMPLICITY Registry. Eur Heart J. 2017;38:93-100.

28. Narkiewicz K, Phillips BG, Kato M, Hering D, Bieniaszewski L, Somers VK. Gender-selective interaction between aging, blood pressure, and sympathetic nerve activity. Hypertension. 2005;45: $522-5$.

29. Julius S, Nesbitt S. Sympathetic overactivity in hypertension a moving target. Am J Hypertens. 1996. https://doi.org/10.1016/ 0895-7061(96)00287-7.

30. Grassi G, Biffi A, Seravalle G, Trevano FQ, Dell'Oro R, Corrao $\mathrm{G}$, et al. Sympathetic neural overdrive in the obese and overweight state. Hypertension. 2019;74:349-58.

31. Joyner MJ, Barnes JN, Hart EC, Wallin BG, Charkoudian N. Neural control of the circulation: How sex and age differences interact in humans. Compr Physiol. 2015;5:193-215.

32. Kario K. Abstract 13787: blood pressure reduction in patients with orthostatic hypertension in the SPYRAL HTN-OFF Med trial. Circulation. 2019;140:A13787-A13787.

33. Magkas N, Tsioufis C, Thomopoulos C, Dilaveris P, Georgio-poulos G, Sanidas E, et al. Orthostatic hypotension: From pathophysiology to clinical applications and therapeutic considerations. J Clin Hypertens. 2019;21:546-54.

34. Kario K. Orthostatic hypertension - a new haemodynamic cardiovascular risk factor. Nat Rev Nephrol. 2013. https://doi.org/10. 1038/nrneph.2013.224.

35. Ewen S, Cremers B, Meyer MR, Donazzan L, Kindermann I, Ukena $\mathrm{C}$, et al. Blood pressure changes after catheter-based renal denervation are related to reductions in total peripheral resistance. J Hypertens. 2015;33:2519-25.

36. Saxena M, Shour T, Shah M, Wolff CB, Julu POO, Kapil V, et al. Attenuation of splanchnic autotransfusion following noninvasive ultrasound renal denervation: a novel marker of procedural success. J Am Heart Assoc. 2018;7. https://doi.org/10.1161/JAHA. 118.009151.

\section{Affiliations}

\section{Manish Saxena $\mathbb{D}^{1} \cdot$ Roland E. Schmieder $\mathbb{D}^{2}$ - Ajay J. Kirtane ${ }^{3}$ - Felix Mahfoud $\mathbb{D}^{4,5}$ - Joost Daemen ${ }^{6}$ Jan Basile ${ }^{7}$. Philipp Lurz ${ }^{8}$ Philippe Gosse $\mathbb{( 1}^{9} \cdot$ Kintur Sanghvi $^{10} \cdot$ Naomi D. L. Fisher $^{11} \cdot$ Lars C. Rump $^{12} \cdot$ Atul Pathak $^{13}$.}


Peter J. Blankestijn ${ }^{14}$ - Anthony Mathur ${ }^{1} \cdot$ Yale Wang $\mathbb{D}^{15} \cdot$ Michael A. Weber $\mathbb{D}^{16} \cdot$ Andrew S. P. Sharp ${ }^{17,18}$.

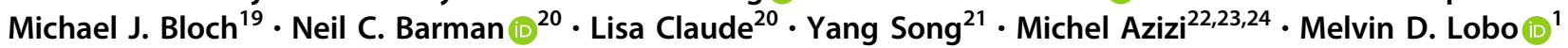

$1 \quad$ Barts NIHR Biomedical Research Centre, William Harvey Research Institute, Queen Mary University of London, London, UK

2 Nephrology and Hypertension, University Hospital Erlangen, Friedrich Alexander University, Erlangen, Germany

3 Columbia University Medical Center/New York-Presbyterian Hospital and the Cardiovascular Research Foundation, New York, NY, USA

4 Institute for Medical Engineering and Science, Massachusetts Institute of Technology, Cambridge, MA, USA

5 Klinik für Innere Medizin III, Saarland University Hospital, Homburg/Saar, Germany

6 Department of Cardiology, Erasmus MC, University Medical Center Rotterdam, Rotterdam, NL, The Netherlands

7 Seinsheimer Cardiovascular Health Program, Medical University of South Carolina, Ralph H Johnson VA Medical Center, Charleston, SC, USA

8 Heart Center Leipzig, University of Leipzig, Leipzig, Germany

9 Hôpital Saint-André - CHU, Bordeaux, France

10 Deborah Heart \& Lung Center, Brown Mills, NJ, USA

11 The Brigham and Women's Hospital, Boston, MA, USA
12 University Clinic Dusseldorf, Dusseldorf, Germany

13 Department of Cardiovascular Medicine, Princess Grace Hospital, Monaco, Monaco

14 University Medical Center Utrecht, Utrecht, The Netherlands

15 Minneapolis Heart Institute, Abbott Northwestern Hospital, Minneapolis, MN, USA

16 Division of Cardiovascular Medicine, State University of New York, Downstate Medical Center, New York, NY, USA

17 University Hospital of Wales, Cardiff, UK

18 University of Exeter, Exeter, UK

19 Department of Medicine, University of Nevada School of Medicine, Vascular Care, Renown Institute of Heart and Vascular Health, Reno, NV, USA

20 ReCor Medical, Inc, Palo Alto, CA, USA

21 Baim Institute for Clinical Research, Boston, MA, USA

22 Université de Paris, Paris, France

23 Hypertension Department and DMU CARTE, AP-HP, Hôpital Européen Georges-Pompidou, Paris, France

24 INSERM, CIC1418, Paris, France 